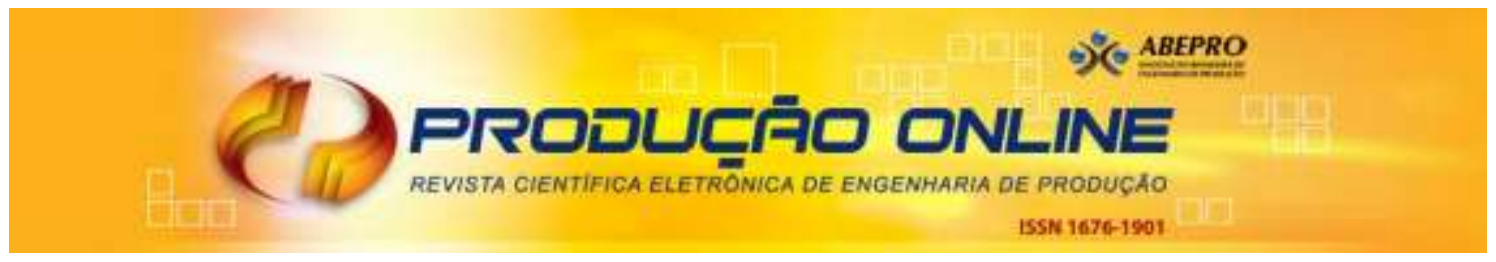

\title{
ANÁLISE DO IMPACTO DA VARIABILIDADE DE FLUXO NO DIMENSIONAMENTO DE KANBANS
}

\section{IMPACT ANALYSIS OF FLOW VARIABILITY IN SIZING KANBANS}

\author{
Isaac Pergher* E-mail: eng.isaac@hotmail.com \\ Luciano Auad da Silva* E-mail: luauadsilva@hotmail.com \\ Diego Augusto de Jesus Pacheco** E-mail: profdajp@gmail.com \\ Guilherme Luis Roehe Vaccaro*E-mail: guilhermev@unisinos.br \\ *Universidade do Vale do Rio dos Sinos (UNISINOS), São Leopoldo, RS \\ **Universidade Federal do Rio Grande do Sul (UFRGS), Porto Alegre, RS
}

\begin{abstract}
Resumo: $O$ objetivo deste artigo é analisar os efeitos da variabilidade de fluxo, preconizada pela Factory Physics, no dimensionamento de Kanban em sistemas produtivos. A variabilidade de fluxo pressupõe que a variabilidade existente nas atividades executadas por um processo é dissipada ao longo de todo o fluxo produtivo do sistema, causando variações no lead time, no nível de estoques em processo e na disponibilidade de equipamentos, entre outros. Para conduzir a investigação, foi criado um modelo didático de simulação computacional por eventos discretos. O modelo proposto visa apresentar os possíveis impactos provocados pela variabilidade de fluxo no sistema produtivo no dimensionamento do número de cartões Kanban, a partir dos resultados fornecidos por dois cenários distintos investigados. Os principais resultados da pesquisa permitem evidenciar que, comparando os dois cenários desenvolvidos para o modelo, a presença de variabilidade no sistema produtivo causou um aumento médio de $32 \%$ no número de cartões Kanban $(p=0,000)$. Isso implica que, em sistemas produtivos reais, o estudo de dimensionamento de Kanbans deveria contemplar as variabilidades individuais das operações, fato por vezes relegado a um pressuposto na formulação da literatura que trata da definição do número de Kanbans, oportunizando assim o desenvolvimento de pesquisas futuras nesse sentido.
\end{abstract}

Palavras-chave: Kanban. Variabilidade de Fluxo. Modelagem. Simulação Computacional. Factory Physics.

\begin{abstract}
The aim of this paper is to analyze the effects of variability flow, advocated by Factory Physics, in sizing Kanban production systems. The variability of flow presupposes that the variability of activities performed by a process is dissipated throughout the productive flow system, causing variations in the lead time, the work-in-process levels and the equipment availability, among others. To conduct the research, we created a didactic model of discrete event computer simulation. The proposed model aims to present the possible impacts caused by the variability flow in a production system regarding the sizing of the number of Kanbans cards, by using the results supplied by two different investigated scenarios. The main results of the research allow concluding that, by comparing the two scenarios developed in the model, the presence of variability in the production system caused an average increase of $32 \%$ in the number of Kanban cards $(p=0,000)$. This implies that, in real productive systems, the study of Kanban sizing should consider the variability of individual operations, a fact often relegated as an assumption in the formulation from classical literature on the definition of the number of Kanbans, thus providing opportunities for the development of future research.
\end{abstract}

Keywords: Kanban, Flow Variability. Modeling. Computer Simulation. Factory Physics. 


\section{INTRODUÇÃO}

A melhoria da eficiência e da eficácia de processos é frequentemente associada à noção de vantagem competitiva (AVELLA-CAMERO et al., 1996; BOYER; LEWIS, 2002; CHRISTIANSEN, 2003; DANGAYACH; DESMUKH, 2001). Essa associação leva organizações a adotarem filosofias que contribuem para a melhoria de seus processos, esperando, assim, resultados como o aumento de flexibilidade, bem como redução de custos e lead time de entrega e serviços, por consequência, melhoria da percepção de qualidade sobre o nível de serviço prestado a seus clientes e usuários (BOYER; LEWIS, 2002; CALIFE; NOGUEIRA; ALVES FILHO, 2010). Uma das metodologias conhecidas no contexto industrial e que contribui com a melhoria dos fatores competitivos devido à busca permanente da eliminação das perdas é o Sistema Toyota de Produção (STP). A gênese dessa metodologia é proporcionar a melhoria da "função processo" melhorando o fluxo da matéria prima no tempo e no espaço, através da compreensão da "função operação" e da eliminação do que não agrega valor. Para tanto, utiliza-se um conjunto de métodos como: Troca Rápida de Ferramentas, Kanban, Poka Yoke, 5S, Padronização da Atividade de Trabalho, Manutenção Preventiva e Células de Manufatura. O STP é sustentado por dois pilares básicos (OHNO, 1997): Autonomação (ou automação com um toque humano), com a função de separar a operação humana da operação de máquinas e equipamentos, e Just-in-time (JIT), focalizando a sincronização das operações e fluxos de materiais na função processo. O uso integrado do STP com abordagens como o Seis Sigma e a Teoria das Restrições, por exemplo, foi discutido no estudo de Pacheco (2012) que abordou o impacto da variabilidade no sistema produtivo. Outra relevante contribuição sobre as perdas produtivas foi feita por Pergher, Rodrigues e Lacerda (2011) que apresentaram o conceito de 'Pergas', o qual faz referência à perda de Ganho Global (Teoria das Restrições) ocorrido pela má definição do mix de produtos.

Além de promover esforços objetivando eliminar perdas, os métodos do STP também buscam a redução da variabilidade, que é um dos princípios do pensamento enxuto (DETTMER, 2001). Paradigmaticamente, o STP preconiza a eliminação da variabilidade de um sistema produtivo, usando métodos de padronização de trabalho 
e operações (ANTUNES et al., 2008; BROWNING; HEATH, 2009). Dessa forma, alicerça-se sobre uma hipótese de que a variabilidade do sistema é desprezível para desenvolver outras abordagens, em especial as associadas ao JIT. Tal estratégia justifica-se, visto que a variabilidade em operações de um sistema produtivo origina os seguintes aspectos (HOPP; SPEARMAN, 2000): (i) diminui o desempenho do sistema produtivo; (ii) gera necessidade de manter estoques para equilibrar as flutuações entre as diferentes fases do processo; (iii) interfere diretamente na qualidade dos produtos; (iv) provoca dificuldades na determinação de prazos de entrega; (v) pode ocasionar finalização do processamento dos produtos antes do prazo de entrega determinado, gerando perdas por superprodução; e (vi) acarreta aumento nos custos da operação do sistema.

Depreende-se, então, que a variabilidade atua de forma significante em fatores competitivos da organização. Diante disso, este trabalho apresenta, por meio de um modelo didático desenvolvido por meio da simulação por eventos discretos, uma análise sobre o impacto da variabilidade de fluxo, como apresentada por Hopp e Spearman (2000), no dimensionamento de um sistema Kanban. Com esse propósito, são avaliados os possíveis impactos no dimensionamento do número de cartões Kanban, com base em dois cenários que abordam diferentes coeficientes de variação, considerando os seguintes indicadores: (i) lead time do contenedor, que nesta pesquisa faz referência ao tempo necessário para produzir um contenedor do Kanban; (ii) número de cartões Kanban; e (iii) estatísticas das filas, que fornece informações estatísticas referentes ao tamanho da fila e tempo de espera do processo, antes de cada estação de trabalho. Uma vez determinado o número de cartões em cada cenário, são analisadas as possíveis diferenças existentes e provocadas pela variabilidade de fluxo.

O restante do artigo é assim organizado: a próxima seção aborda os conceitos teóricos sobre a variabilidade segundo a Factory Physics e as particularidades do sistema Kanban. Em seguida é apresentado a metodologia utilizada no desenvolvimento da pesquisa. O desenvolvimento e as premissas do modelo didático de simulação computacional são explicados na seção quatro. Por fim, são analisados os resultados obtidos quanto às diferenças no dimensionamento do número de cartões Kanban entre os dois cenários criados. O artigo finaliza com a apresentação das conclusões do estudo. 


\section{REFERENCIAL TEÓRICO}

\subsection{Particularidades do Sistema Kanban}

O Kanban é uma técnica que visa controlar o desempenho dos materiais da produção (PETTERSEN; SEGERSTEDT, 2009). Para Sandrini e Mesquita (2008), esta técnica é utilizada para controlar a produção no chão de fábrica e limitar a quantidade de material em processo. Slack, Chambers e Johnston (2009) definem o Kanban como um método de operacionalizar o sistema de planejamento e controle puxado. Segundo Antunes et al. (2008), essa técnica pode ser entendida como uma 'ferramenta' de programação e controle da produção, a qual simplifica os sistemas os tradicionais de controle da produção (ordens de fabricação, montagem e compras) na medida em que, adotando um controle visual do material que circula na fabrica, elimina toda a circulação de papéis na estrutura de fabricação.

Existem diferentes tipos de sistemas Kanban: (i) de transporte, com o objetivo de informar o estágio anterior que determinado material pode ser retirado do estoque e transferido para um destino específico; (ii) de produção, com a função de sinalizar o início de produção do item descriminado no cartão; e (iii) de fornecimento, com a função de informar o fornecedor sobre a necessidade de envio de materiais ou componentes ao sistema produtivo principal. Vantagens do uso dessa abordagem referem-se à redução dos custos de inventário, armazenagem, entregas e o aumento da flexibilidade (RABBANI; LAYEGH; EBRAHIM, 2009).

Para utilizar o Kanban como sistema de auxílio à gestão do fluxo de materiais, primeiramente deve-se atender a seus pressupostos de pré-implantação. Para Sandanayake, Oduoza e Proverbs (2008), a falta de qualidade, a variabilidade no desempenho do operador e a inconsistência na distribuição de tarefas, comprometem diretamente a eficiência do sistema JIT. Dentre esses, segundo os autores, assegurar a qualidade dos diferentes níveis de fabricação é elemento central para a produção sincronizada JIT. Além disso, dada sua suscetibilidade à ação da variabilidade sobre o sistema, há necessidade de estabelecer nivelamento de demanda e padronização de tempos e operações. Isto por que o sistema Kanban 
não opera de forma satisfatória em ambientes instáveis, conforme (SHAHABUDEEN; SIVAKUMAR, 2008). Por fim, o estudo de Marodin, Eckert e Saurin (2012) verificou o impacto da definição correta do Kanban na logística interna Lean e no fluxo de materiais, destacando-o como um aspecto relevante no sistema produtivo.

Com relação às incertezas (ocasionadas pela variação dos tempos ou falta de padronização) de um processo produtivo, o Kanban pode operar com pequenos níveis de estoque, quando as condições de variabilidade são baixas (SUWANRUJl; ENNS, 2006). Conforme Antunes (1998) quanto maior a variabilidade do processo maior será a necessidade da utilização de estoques. Além do alto nível de estoque, a variabilidade pode causar filas, atrasos e aumentar o estoque em processo (BONVIK; GERSHWIN; TROXEL, 1995). Melhorar o desempenho dos estoques resulta em importantes melhorias no fluxo de caixa e em aumentos da lucratividade (PONTES; PALMA; PORTO, 2008).

Uma vez atendidos os pressupostos anteriormente mencionados, é possível calcular o número de cartões Kanban a serem utilizados no sistema. A literatura apresenta tipicamente abordagens de cálculo desenvolvidas a partir de padrões determinísticos, similares ao preconizado por Shingo (1996) e também, modelos que incorporam técnicas derivadas da Pesquisa Operacional. Uma das maneiras de calcular o número de cartões Kanban é apresentado por Corrêa et al. (2001), na Equação 1:

$$
X=\frac{D\left(T_{e}+T_{P}\right)(1+F)}{C}
$$

Onde: $X=$ número total de cartões Kanban; $D=$ demanda do centro consumidor por unidade de tempo; $T_{e}=$ tempo de espera do lote no centro produtor; $T_{p}=$ tempo de processamento do lote no centro produtor; $C=$ tamanho do lote ou capacidade do contêiner (peças por Kanban); $F=$ fator de segurança.

Observa-se que os elementos promotores de variabilidade não são tipicamente considerados nas abordagens de cálculo de Kanban, uma vez que se aceite que o pressuposto de minimização dos fatores de variabilidade foi atendido anteriormente a esta etapa. Ainda que essa abordagem seja respaldada pela literatura (LAGE Jr; GODINHO FILHO, 2008), e deva ser considerada como primeira alternativa na implementação de Kanban, há que se considerar que: (i) sistemas produtivos podem estar sujeitos a fontes de variação não controláveis ou cujo 
controle possa ser excessivamente exaustivo ou custoso (LAGE Jr; GODINHO FILHO, 2008; WHITE; PRYBUTOK, 2001); (ii) em certas situações, os efeitos da padronização de tempos e operações podem deteriorar-se ao longo do tempo (TUBINO; LEMOS, 1999; LAGE Jr; GODINHO FILHO, 2008; FERNANDES et al., 2001); e (iii) a implementação de sistemas Kanban pode ser inadequada, desconsiderando a relevância da eliminação da variabilidade e seus efeitos no fluxo do processo de produção (TUBINO; LEMOS, 1999; LAGE Jr; GODINHO FILHO, 2008; FERNANDES et al., 2001; FERNANDES; GODINHO FILHO, 2007). Em todos os casos citados, justifica-se compreender a influência da variabilidade sobre a especificação do número de cartões Kanban, como proposto neste artigo. Para tanto, apresentam-se, na seção seguinte, informações sobre o conceito de Variabilidade e seus efeitos, conforme a visão da Factory Physics.

\subsection{A Variabilidade segundo a Factory Physics}

Hopp e Spearman (2000) descrevem, sob a designação Factory Physics, um conjunto de leis e equações que podem ser utilizadas para promover o entendimento aprofundado dos sistemas produtivos. No que tange à temática da variabilidade, Hopp e Spearman (2000) definem-na como a ausência de uniformidade em grupos de dados. Neste sentido, segundo os autores, a variabilidade pode ser classificada em duas maneiras: variabilidade 'Boa' ou 'Ruim', sendo os conceitos de cada tipo, apresentados no Quadro 1. Tipicamente, o conceito de variabilidade 'Boa' pode ser associado à promoção de diversidade e de variedade, podendo levar a vantagens competitivas, discutidas pelos autores em seu livro. Já a variabilidade 'Ruim' pode ser associada a efeitos danosos ao desempenho ou efetividade do sistema de produção, levando a retrabalhos ou outros tipos de perdas.

Quadro 1 - Definição da variabilidade Boa e variabilidade Ruim

\begin{tabular}{|c|c|c|c|}
\hline \multicolumn{2}{|c|}{ Variabilidade Ruim } & \multicolumn{2}{|c|}{ Variabilidade Boa } \\
\hline Causa & Exemplo & Causa & Exemplo \\
\hline Paradas planejadas & Setups & Variedade de produtos & $\begin{array}{l}\text { GM nas décadas de } \\
1930 \text { e } 1940\end{array}$ \\
\hline $\begin{array}{l}\text { Paradas não } \\
\text { planejadas }\end{array}$ & $\begin{array}{l}\text { Falhas de } \\
\text { equipamento }\end{array}$ & $\begin{array}{l}\text { Mudanças } \\
\text { tecnológicas }\end{array}$ & $\begin{array}{l}\text { Intel nas décadas de } \\
1980 \text { e } 1990\end{array}$ \\
\hline $\begin{array}{l}\text { Problemas de } \\
\text { qualidade }\end{array}$ & $\begin{array}{l}\text { Perda de rendimento } \\
\text { ou retrabalhos }\end{array}$ & $\begin{array}{l}\text { Variabilidade da } \\
\text { demanda }\end{array}$ & Empresa Jiffy Lube \\
\hline Variações dos & Diferenças de & & \\
\hline
\end{tabular}




\begin{tabular}{|l|l|}
\hline operadores & habilidades \\
\hline Projeto inadequado & $\begin{array}{l}\text { Alterações de } \\
\text { engenharia }\end{array}$ \\
\hline
\end{tabular}

Fonte: adaptado de Hopp e Spearman (2000).

Conforme Hopp e Spearman (2000), as seguintes equações podem ser empregadas para analisar a variabilidade existente em um determinado processo. Cabe ressaltar que a métricas apresentadas neste trabalho, referem-se a paradas acidentais não controláveis, como por exemplo, a falta de energia elétrica.

a) Coeficiente de variação do tempo de processamento natural $\left(C_{0}\right)$ : tem como objetivo avaliar a variabilidade natural existente no processo (Equação 2). A variabilidade natural é inerente aos tempos de processo naturais, excluindo-se paradas aleatórias, setups, ou quaisquer outras influências externas. Esse tipo de variabilidade é motivado por fatores não controláveis, intrínsecos a cada sistema, referindo-se a flutuações nos tempos de processamento, causados por diferenças nas habilidades dos operadores, máquinas e materiais.

$$
C_{0}=\frac{\sigma_{0}}{T_{0}}
$$

Onde: $\sigma_{0}$ é o desvio padrão natural e $T_{0}$ é a média natural do tempo de processamento.

Ao calcular $\left(C_{0}\right)$, é possível comparar o valor obtido, com um índice padrão, apresentado no Quadro 2, o qual, segundo os Hopp e Spearman (2000), permite definir a classe da variabilidade existente no ambiente estudado.

Quadro 2 - Classes de variabilidade

\begin{tabular}{|c|c|l|}
\hline Classes & Coeficiente de variação & \multicolumn{1}{|c|}{ Típicas situações } \\
\hline Baixa & $C_{0}<0.75$ & Tempos de processo sem paradas \\
\hline Moderada & $0.75 \leq C_{0}<1.33$ & $\begin{array}{l}\text { Tempos de processos com ajustes rápidos } \\
\text { (exemplo: setups) }\end{array}$ \\
\hline Alta & $C_{0} \geq 1.33$ & $\begin{array}{l}\text { Tempos de processos com longas paradas } \\
\text { (exemplo: quebra de máquinas) }\end{array}$ \\
\hline
\end{tabular}

Fonte: Hopp e Spearman (2000)

b) Coeficiente de variação do tempo de processamento natural ao quadrado $\left(C^{2}{ }_{0}\right)$ : segundo Hopp e Spearman (2000), o uso da variância em muitos casos é a forma mais conveniente de representar a variabilidade do processo. Por esse motivo, a Equação 3 pode ser utilizada para comparar os níveis de variabilidade em diferentes sistemas. 


$$
C_{0}^{2}=\frac{\square \sigma_{0}}{\square T_{0}} \square
$$

c) Disponibilidade $(A)$ : refere-se à disponibilidade de um equipamento, em termos de operabilidade sem interrupções provocadas por problemas de manutenção. É expresso como na Equação 4, identicamente aos conceitos pertencentes à disciplina de Confiabilidade e Manutenção de Equipamentos.

$$
A=\frac{M T T F}{M T T F+M T T R}
$$

Onde: MTTF (Mean Time to Failure) representa o tempo médio que o equipamento opera até falhar, e MTTR (Mean Time to Repair) representa o valor temporal médio gasto para consertar o equipamento.

d) Coeficiente de variação dos tempos de reparo $\left(C_{r}\right)$ : refere-se ao coeficiente de variação dos valores temporais gastos para colocar o equipamento danificado em condições de uso. O pressuposto da Equação 5 é a ocorrência de distribuições de probabilidade iguais entre as métricas $\sigma_{R}$ e MTTF.

$$
C_{r}=\frac{\sigma_{R}}{M T T F}
$$

Onde: $\sigma_{R}$ é o desvio padrão amostral dos dados referentes ao MTTF.

Além desses indicadores, Hopp e Spearman (2000) também consideram relevante a mensuração do tempo de processamento efetivo, que diz respeito ao tempo total consumido por uma operação em uma estação de trabalho, considerando questões referentes à disponibilidade $(A)$. Este fator é dividido em dois índices, descritos a seguir:

e) Tempo médio efetivo de processo $\left(T_{e}\right)$ : refere-se ao tempo do processo, porém considerando a disponibilidade do equipamento, a qual atua inflacionando o tempo médio natural de processamento (Equação 6).

$$
T_{e}=\frac{T_{0}}{A}
$$

f) Coeficiente de variação do tempo de processamento efetivo ao quadrado $\left(C^{2}{ }_{e}\right)$ : este coeficiente ilustra o impacto causado por questões relativas à Confiabilidade de equipamentos sobre o tempo de processamento (Equação 7).

$$
C_{e}^{2}=C_{0}^{2}+\left(1+C_{r}^{2}\right) A(1-A) \frac{M T T R}{T_{0}}
$$


As equações 2 a 7 permitem analisar a influência da variabilidade nos tempos de produção de uma estação de trabalho específica. Em particular, observa-se, pela Equação (7), que equipamentos com alta disponibilidade tendem a sofrer menores efeitos inflacionários das componentes relativas à aspectos de manutenção, coerentemente com o preconizado pelo STP, que orienta a utilização de equipamentos simples e de fácil manutenção em lugar de máquinas monumento (SHINGO, 1996), as quais tendem a ter tempos de reparo maiores e modos de falha mais complexos, elevando o valor de $\mathrm{C}^{2}$.

No que tange à análise da variabilidade em sistemas produtivos formados por diferentes estações de trabalho, Hopp e Spearman (2000) descrevem o que denominam Lei da Variabilidade o fato de que o aumento da variabilidade sempre degrada o desempenho do sistema de produção. O aumento da variação nos tempos operacionais pode ser originado por diversos fatores, tais como falta de treinamento, ausência de padronização de atividades, políticas de manutenção inadequadas, entre outros. Neste sentido, quando ocorre o aumento da variabilidade na estação antecedente, este evento pode provocar discrepâncias nas taxas de abastecimento da estação subsequente, gerando impactos no seu desempenho, e assim sucessivamente. Este fato é denominado, pelos autores, Variabilidade de Fluxo. Para avaliar este tipo de variabilidade, Hopp e Spearman (2000) descrevem um conjunto de métricas apresentadas na Figura 1, a qual representa um sistema produtivo simplificado, ilustrando o fluxo de materiais entre duas estações de trabalho.

Figura 1 - Variabilidade entre estações. Fonte: adaptado de Hopp e Spearman (2000).

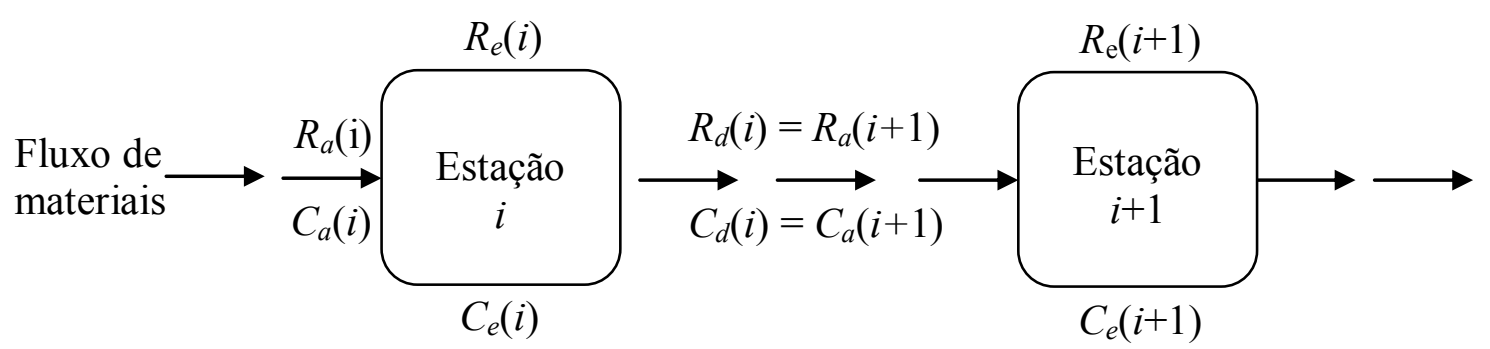

O fluxo apresentado na Figura 1 pode ser compreendido em termos de taxas de entrada e saída dos materiais nas estações de trabalho. Com este enfoque, ao observar a estação $i$, o valor de $R_{a}(i)$ ilustra a taxa de chegada dos itens a serem 
processados, que são enviados pelo processo antecessor. Assumindo-se uma distribuição exponencial, tem-se:

$$
R_{a}(i)=\frac{1}{t_{a}(i)}
$$

Onde: $t_{a}(i)$ é o tempo médio entre chegadas na estação $i$.

Referente à taxa de chegada $R_{a}(i)$, é necessário calcular o coeficiente de variação dos tempos entre chegadas, $C_{a}(i)$ :

$$
C_{a}(i)=\frac{\sigma_{a}(i)}{t_{a}(i)}
$$

Onde: $\sigma_{a}(i)$ representa o desvio padrão dos tempos entre chegadas da estação $i$.

Ao iniciar o processamento, a taxa de saída na estação $i$ dependerá da taxa de capacidade efetiva nessa mesma estação, dado por $R_{e}(i)$. A Equação 10 apresenta o modelo para cálculo deste índice. No entanto, para que uma estação possa dar conta das chegadas, é preciso que a capacidade seja maior do que a taxa de chegada, isto é $R_{e}>R_{a}$.

$$
R_{e}(i)=\frac{M(i)}{T_{e}(i)}
$$

Onde: $M(i)$ representa o número de recursos produtivos, pertencentes à estação $i$.

Ainda conforme a Figura 1, ao finalizar o processamento do item na estação $i$, este é enviado para a estação i+1. O envio de itens já processados é dado pela taxa de saída da estação $i$, representado por $R_{d}(i)$. No caso representado pela Figura 1 , a taxa de saída da estação $i$ é igual à taxa de chegada na estação $i+1$, dado por $R_{a}(i+1)$. Para calcular a taxa da saída da estação $i$, utiliza-se a Equação 11.

$$
R_{d}(i)=R_{a}(i+1)=\frac{1}{T_{d}(i)}
$$

Onde: $T_{d}(i)$ é a média dos tempos entre saídas da estação $i$.

Para calcular o coeficiente de variação dos tempos entre chegadas da estação $i+1$, que, no caso descrito, é igual ao coeficiente de variação dos tempos entre saídas da estação $i$, é necessário considerar os seguintes elementos referentes à estação $i$ : a utilização $U(i)$, o coeficiente de variação dos tempos entre chegadas $C_{a}(i)$, e o coeficiente de variação do tempo de processamento efetivo ao quadrado $C^{2}(i)$. A Equação 12 ilustra a forma de calcular a utilização $(U)$ da estação $i$, conceituada como a fração do tempo em que a máquina não permaneceu ociosa 
por falta de envio de materiais para serem processados. Ao utilizar a Equação 12 em estações constituídas por diversos recursos produtivos $(M>1)$, é necessário que estes recursos possuam características semelhantes.

$$
U(i)=\frac{R_{a}(i) \square T_{e}(i)}{M}
$$

Por fim, a Equação 13 representa o coeficiente de variação dos tempos entre chegadas da estação $i+1$, considerando estações de trabalho que possuem um único recurso produtivo.

$$
C_{a}^{2}(i+1)=U_{(i)}^{2} C_{e}^{2}(i)+\left(1-U_{(i)}^{2}\right) C_{a}^{2}(i)
$$

Ao finalizar o processamento na estação $i+1$, a taxa de saída da estação $i+1$, representado por $R_{d}(i+1)$ e o coeficiente de variação dos tempos entre saídas $C_{d}(i+1)$ dependem da taxa de capacidade efetiva $R_{e}(i+1)$, da utilização $U(i+1)$, do coeficiente de variação dos tempos entre chegadas $C_{a}(i+1)$ e do coeficiente de variação do tempo de processamento efetivo ao quadrado $C^{2}{ }_{e}^{(i+1)}$, e assim sucessivamente.

É possível observar, com base nas equações 8 a 13, que a variabilidade ocorrida em uma determinada estação de trabalho é propagada ao longo do sistema produtivo, devido à conexão intrínseca existente entre as taxas de entrada e saída e a capacidade efetiva das e entre as estações de trabalho. Considerando constante a taxa de chegada em uma estação ' $i$, assumida com grau de variabilidade alto no processo, podem ocorrer as seguintes situações na estação ' $i+1$ ', assumida com grau de variabilidade mais baixo (Quadro 3).

Quadro 3 - Prováveis consequências da variabilidade de fluxo

\begin{tabular}{|c|c|c|}
\hline \multicolumn{2}{|c|}{ Estação ‘' } & Estação ‘i+1' \\
\hline $\begin{array}{c}\text { Grau de variabilidade alto } \\
\begin{array}{c}\text { estiver em valores baixos da } \\
\text { distribuição de probabilidade }\end{array}\end{array}$ & $\begin{array}{c}\text { Prováveis } \\
\text { consequências }\end{array}$ & Grau de variabilidade baixo \\
\hline $\begin{array}{c}\text { Quando o tempo de processamento a taxa de } \\
\text { atingir o mínimo da distribuição de } \\
\text { probabilidade }\end{array}$ & $\begin{array}{c}\text { É atingido o máximo } \\
\text { da taxa de saída de } \\
\text { materiais }\end{array}$ & $\begin{array}{c}\text { Maior taxa de incremento no } \\
\text { tamanho da fila e no tempo de } \\
\text { espera de processo }\end{array}$ \\
\hline $\begin{array}{c}\text { Quando o tempo de processamento } \\
\text { estiver em valores altos da } \\
\text { distribuição de probabilidade }\end{array}$ & $\begin{array}{c}\text { Diminuição da taxa de } \\
\text { saída de materiais }\end{array}$ & $\begin{array}{c}\text { Redução no tamanho da fila e do } \\
\text { tempo de espera de processo. } \\
\text { Pode ocorrer ociosidade }\end{array}$ \\
\hline $\begin{array}{c}\text { Quando o tempo de processamento } \\
\text { atingir o máximo da distribuição de } \\
\text { probabilidade }\end{array}$ & $\begin{array}{c}\text { É atingido o mínimo da } \\
\text { taxa de saída de } \\
\text { materiais }\end{array}$ & $\begin{array}{c}\text { Maior taxa de incremento de } \\
\text { ociosidade do equipamento }\end{array}$ \\
\hline
\end{tabular}

Fonte: autores (2013) 
No que tange ao cálculo das filas, Hopp e Spearman (2000) apresentam os modelos $M / M / 1$ e VUT. No modelo $M / M / 1$ é necessário atender os seguintes pressupostos: tempos de chegada exponenciais, máquina única na estação de trabalho com tempos de processamento exponenciais, protocolo de atendimento do tipo primeiro a chegar, primeiro a ser atendido e um espaço ilimitado de tamanho de fila. O modelo VUT não necessita desses pressupostos, no entanto, fornece o tempo médio de lead time na fila e não mensura o exato desempenho da fila.

Entretanto, pode se tornar difícil a análise da variabilidade de fluxo, utilizando as equações anteriormente abordadas, em termos de determinação de estatísticas descritivas como: média, desvio padrão, máximo e mínimo valor, quando as taxas de chegada, processamento e saída, tempos médios até a falha (MTBF) e os tempos médios de reparo (MTTF) seguem diferentes distribuições probabilísticas, não atendendo assim aos pressupostos anteriormente relatados. A complexidade de cálculo pode aumentar conforme a característica dos indicadores que se deseja monitorar, bem como a configuração do sistema produtivo estudado, dificultando o planejamento e a tomada de decisão na prática gerencial. Neste sentido, o uso da simulação computacional pode contribuir de forma significativa para estudar a variabilidade de fluxo, devido à possibilidade de considerar no estudo, a falta de atendimento dos pressupostos necessários na análise das filas e também o uso de diferentes distribuições de probabilidade existentes nas operações.

\section{PROCEDIMENTOS METODOLÓGICOS}

A pesquisa foi desenvolvida com base no método de simulação computacional, como preconizado por Law e Kelton (2000). Em se tratando de um estudo conceitual, inicialmente realizou-se o levantamento do referencial necessário para a realização da pesquisa. Posteriormente, desenvolveu-se o modelo base de simulação, integrando os indicadores necessários para a avaliação dos cenários de análise propostos.

O método apresentado na Equação 1 foi usado na parametrização do modelo didático de simulação para definir o número de cartões Kanban, considerando as particularidades propostas pelos cenários A e B. Para fins de delimitação de escopo, foram analisadas as seguintes questões, referentes à variabilidade indesejada ou 
'Ruim': (i) paradas planejadas; (ii) paradas não planejadas; e (iii) variações dos operadores.

O tempo de transportar entre estações de trabalho e do contenedor não foi considerado no modelo didático de análise. Essa decisão justifica-se por representar um contexto de menor variabilidade potencial do que outro em que os tempos de transporte fossem significantes.

Os cenários de análise propostos foram configurados de modo a possibilitar a apresentação dos possíveis impactos gerados pela variabilidade no fluxo produtivo, no que faz referência ao número de cartões Kanban, lead time do contenedor e estatísticas das filas. Neste âmbito foram desenvolvidos os cenários $A$ e $B$ à luz do grau de variabilidade. $O$ cenário $A$ representa a ausência de variabilidade nos tempos de processamento, preparação (setup), MTBF e MTTF. Entretanto o cenário B apresenta um grau moderado de variabilidade $C_{0}<1.33$ (HOPP; SPEARMAN, 2000) para esses elementos.

Para fins de análise dos resultados, foi usado o teste $\mathrm{T}$, considerando-se um nível de significância de $10 \%$ e um poder de $75 \%$ com sensibilidade de deteç̧ão de diferenças superiores a meio desvio-padrão. $O$ teste $T$ examina a diferença entre grupos e examina estatisticamente a igualdade das médias de dois grupos de dados (HAIR et al., 2009). Esse teste preconiza como hipótese nula $\left(\mathrm{H}_{0}\right)$ a igualdade entre as médias e como hipótese alternativa a diferença, sendo considerada verdadeira a hipótese alternativa (e rejeitada $\mathrm{H}_{0}$ ) em situações em que o resultado do teste $\mathrm{T}$ é menor que o nível de significância previamente estabelecido. $O$ teste $T$ requer que as distribuições das variáveis de saída sejam normais, fato que pode ser verificado pelo uso do Teste de Kolmogorov-Smirnov. Outro teste comumente referido para verificar a normalidade das distribuições é o teste de Anderson-Darling. Maiores detalhes podem ser obtidos em Law e Kelton (2000) e em Hair et al. (2009).

O modelo de simulação foi então desenvolvido e foi realizado um total de 30 rodadas consecutivas para o cenário $B$, dado que o cenário $A$ é determinístico. Isto representa 9 dias de trabalho de 3 turnos cada, realizando a coleta das informações referentes aos indicadores, bem como, as variáveis temporais dependentes intrínsecas a cada estação de trabalho, as quais serão apresentadas a seguir. 0 modelo de simulação foi desenvolvido no software Micro Saint 3.0 e as análises foram realizadas no software Minitab 16. 


\subsection{Descrição do modelo de análise}

O modelo didático proposto para a análise tem como meta representar um sistema produtivo que foi efetivado por meio de informações hipotéticas, avaliar possíveis impactos da variabilidade de fluxo no dimensionamento do sistema Kanban. Na Figura 2 é apresentada a configuração do modelo desenvolvido. $\mathrm{O}$ modelo é constituído de 3 estações de trabalho posicionadas em série. Foi definido que é necessária a realização da operação de setup, antes de iniciar o processamento. Cada estação é composta por um equipamento e um operador. As principais diferenças entre as estações de trabalho nos dois cenários $A$ e $B$ propostos, referem-se aos valores de tempo de processamento natural e à disponibilidade da estação 3, conforme evidenciado nas Tabelas 1 e 2 .

Figura 2 - Configuração do sistema produtivo

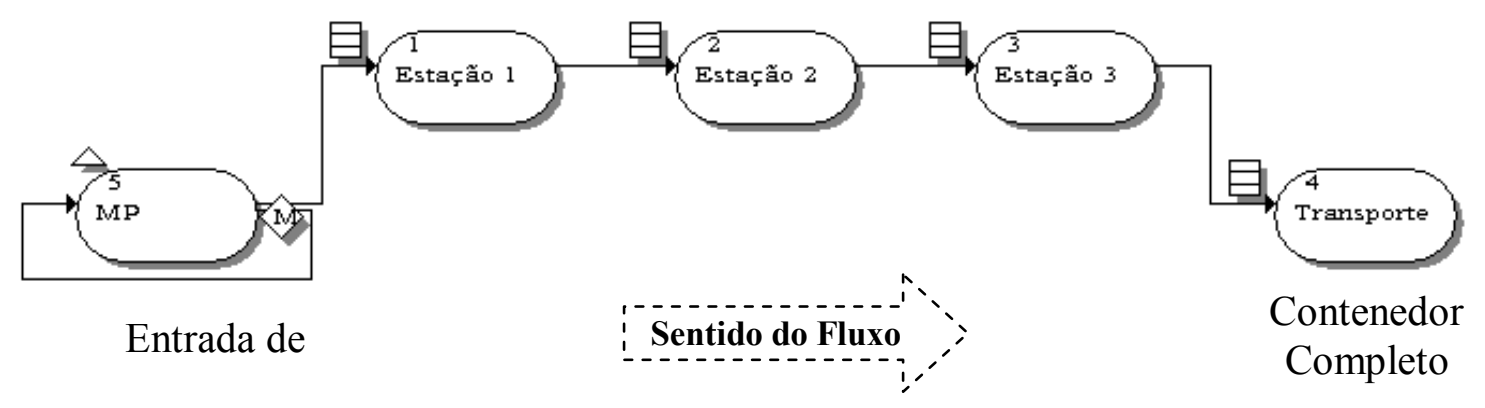

Fonte: autores

Tabela 1 - Dados de entrada para cada cenário. (tempos em minutos)

\begin{tabular}{clccc}
\hline Cenário & \multicolumn{1}{c}{ Parâmetros } & Estação 1 & Estação 2 & Estação 3 \\
\hline & $\begin{array}{l}\text { Média dos tempos de processamento } \\
\text { natural }\end{array}$ & 22 & 42 & 28 \\
A $\quad \begin{array}{l}\text { Desvio padrão dos tempos de } \\
\text { processamento natural }\end{array}$ & 0 & 0 & 0 \\
$\begin{array}{l}\text { Coeficiente de variação do tempo de } \\
\text { processamento natural }\end{array}$ & 0 & 0 & 0 \\
$\begin{array}{l}\text { Média dos tempos de processamento } \\
\text { natural }\end{array}$ & 22 & 42 & 28 \\
Besvio padrão dos tempos de & $\begin{array}{l}\text { processamento natural } \\
\text { Coeficiente de variação do tempo de } \\
\text { processamento natural } \\
\text { Distribuição de probabilidade }\end{array}$ & 0.88 & 0.85 & 28 \\
& Lognormal & Normal & Exponencial \\
\hline
\end{tabular}

Fonte: autores (2013). 
O diagrama apresentado na Figura 2 simula a produção de um único componente, sendo necessária uma quantidade de 10 itens para completar um contenedor, que possui uma demanda semanal determinística igual de 4 contenedores. Neste sentido, compete salientar que o indicador lead time do contenedor mede o tempo para que o processo gasta para produzir um contenedor do Kanban (com 10 itens) à luz dos cenários em análise.

O processo funciona da seguinte maneira: primeiramente é realizada a operação de setup, que ocorre de forma simultânea em cada estação. Uma vez finalizado o setup na estação 1, é iniciada a atividade independente da conclusão da operação setup nas outras estações de trabalho. Na estação 1 considera-se disponível o estoque de matéria-prima a ser utilizado, não havendo consumo de tempo na sua alimentação. Ao finalizar o processamento de um item na estação 1 , este é imediatamente enviado para a estação 2. Quando o item chega nesta estação, são realizadas as atividades que fazem parte do seu plano de trabalho. Ao concluir o processamento na estação 2 , o item é enviado para a estação 3 , onde é realizada as atividades pertinentes a esta fase. Por fim, ao concluir o processamento na estação 3, o item permanece em "espera do lote" até que a quantidade necessária para completar um contenedor seja atendida. Uma vez completado o contenedor, este é transportado para o local do Kanban.

Na Tabela 2 são apresentados os tempos relativos à operação setup, tempo médio entre falhas MTBF e o tempo médio para reparo MTTR. Durante a modelagem, os seguintes pressupostos foram assumidos: (i) o lote de transferência de materiais entre as estações é unitário, ou seja, uma vez finalizado o processamento em qualquer estação, no mesmo instante o item é enviado para a estação subsequente; (ii) no momento inicial da simulação, a linha de processamento apresentada na Figura 2 está vazia e os operadores estão disponíveis para a realização do setup de maneira simultânea em cada estação; (iii) pode ocorrer a formação de filas nas estações 2 e 3, motivadas por diferenças entre os tempos de processamento natural de cada estação, pela disponibilidade da estação 3 e pelo coeficiente de variabilidade inerente a cada estação; (iv) nenhuma condição que limite o fluxo de materiais entre as diferentes etapas do sistema produtivo foi desenvolvida; (v) a taxa de saída da estação 1 é igual a taxa de chegada da estação 2 e a taxa de saída da estação 2 é igual a taxa de chegada da 
estação 3; e (vi) a parada da estação 3 por problemas de confiabilidade funciona da seguinte maneira - quando o relógio do simulador atinge o valor estipulado no MTBF, a estação para e imediatamente inicia a contagem do tempo de reparo, denominado por MTTF. Se o valor temporal do MTBF coincidir com o processamento de algum item, primeiramente é finalizado o processamento e após é iniciada a contagem do tempo de reparo.

Tabela 2 - Dados de entrada para cada cenário. (tempos em minutos)

\begin{tabular}{ccccccc}
\hline \multirow{2}{*}{ Cenário } & Parâmetros & \multicolumn{2}{c}{ Tempo gasto com Setup } & \multicolumn{2}{c}{ MTBF } & MTTR \\
& & Estação & Estação & & Estação 3 \\
\hline \multirow{3}{*}{ A } & Média & 5 & 2 & 7 & 70 & 35 \\
& Desvio Padrão & 0 & 0 & 0 & 0 & 0 \\
& Coeficiente de Variação & 0 & 0 & 0 & 0 & 0 \\
B & Média & 5 & 2 & 7 & 70 & 35 \\
& Desvio Padrão & 5 & 2 & 7 & 70 & 26 \\
& Coeficiente de Variação & 1 & 1 & 1 & 1 & 0.742 \\
& Distribuição de & & Exponencial & & Lognormal
\end{tabular}

Fonte: autores (2013).

A próxima seção apresenta os resultados obtidos e analisa os mesmos à luz do referencial anteriormente apresentado.

\section{DISCUSSÃO DOS RESULTADOS}

A análise dos resultados é feita em termos de tempos de início de processamento e saída da estação. Na variável dependente 'tempo de início do processamento' são ilustrados os valores temporais de início do processamento de cada item na estação de trabalho. Já o 'tempo de saída' mostra o tempo em que o item sai da estação. Cabe ressaltar que o tempo de chegada aqui mencionado refere-se à pronta disposição do item na estação, para que o mesmo possa ser processado quando o recurso produtivo tiver capacidade disponível.

As informações pertencentes ao cenário A são apresentadas de forma ilustrativa na Tabela 3. A estação 1 inicia o processamento do primeiro item no tempo 5 minutos (tempo de finalizar a operação setup na estação 1). Para um tempo de processamento natural igual a 22 minutos, o primeiro item deixa a estação de trabalho no tempo 27 minutos, o segundo item no tempo 49 min, que é dado pela soma do tempo de saída da primeira peça (27 minutos) e o tempo de 
processamento natural dessa estação (22 minutos) e assim sucessivamente até a saída do décimo item. A estação 2 inicia o processamento do primeiro item no mesmo instante em que este deixa a estação 1 , pois estava ociosa. $O$ valor do tempo de setup da estação 2 é igual a 2 minutos e a chegada de trabalho na estação ocorreu no tempo 27 minutos.

Tabela 3 - Tempos de início e Resultados obtidos no cenário A. (Tempos em minutos)

\begin{tabular}{|c|c|c|c|c|c|c|}
\hline \multirow[b]{2}{*}{ Item } & \multicolumn{2}{|c|}{ Estação 1} & \multicolumn{2}{|c|}{ Estação 2} & \multicolumn{2}{|c|}{ Estação 3} \\
\hline & $\begin{array}{c}\text { Tempo de } \\
\text { início do } \\
\text { processamento }\end{array}$ & $\begin{array}{l}\text { Tempo de } \\
\text { saída }\end{array}$ & $\begin{array}{c}\text { Tempo de } \\
\text { início do } \\
\text { processamento }\end{array}$ & $\begin{array}{l}\text { Tempo de } \\
\text { saída }\end{array}$ & $\begin{array}{c}\text { Tempo de } \\
\text { início do } \\
\text { processamento }\end{array}$ & $\begin{array}{l}\text { Tempo de } \\
\text { saída }\end{array}$ \\
\hline 1 & 5 & 27 & 27 & 69 & 69 & 97 \\
\hline 2 & 27 & 49 & 69 & 111 & 132 & 160 \\
\hline 3 & 49 & 71 & 111 & 153 & 160 & 188 \\
\hline 4 & 71 & 93 & 153 & 195 & 195 & 223 \\
\hline 5 & 93 & 115 & 195 & 237 & 258 & 286 \\
\hline 6 & 115 & 137 & 237 & 279 & 286 & 314 \\
\hline 7 & 137 & 159 & 279 & 321 & 321 & 349 \\
\hline 8 & 159 & 181 & 321 & 363 & 384 & 412 \\
\hline 9 & 181 & 203 & 363 & 405 & 412 & 440 \\
\hline 10 & 203 & 225 & 405 & 447 & 447 & 475 \\
\hline
\end{tabular}

Fonte: autores (2013).

O tempo de processamento natural da estação 2, conforme apresentado na Tabela 1 é de 42 minutos, portanto, o primeiro item sai da estação 2 no tempo 69 minutos. Já o segundo item foi enviado para a estação 2 (tempo de chegada) no tempo 49 minutos, mas o processamento do segundo item na estação 2 foi iniciado somente no tempo 69 minutos, permanecendo em espera de processo durante 20 minutos. Isto ocorreu por causa das diferenças entre os tempos de processamento naturais das estações 1 e 2.

Essa disparidade entre os tempos de processamento causa aumento crescente no tempo de espera de processo. O primeiro item não aguardou na fila porque o recurso estava ocioso. Já o segundo item, aguarda 20 minutos para iniciar o processamento, o terceiro e quarto item, aguardam 40 e 60 minutos, respectivamente, e assim até o décimo item, que espera 180 minutos na fila da estação 2 até ocorrer seu processamento. Quando é finalizado o processamento de um item na estação 2, este é direcionado para a estação 3, que também permanecia ociosa aguardando a chegada de trabalho. A estação 3 finalizou operação de setup no tempo 7 minutos e permaneceu ociosa até o tempo 69 minutos, totalizando 62 
minutos de ociosidade. Nesta estação, ocorrem problemas de disponibilidade de equipamentos, conforme apresentado anteriormente na Tabela 2.

$\mathrm{Na}$ Tabela 3, observa-se que o primeiro item sai da estação 2 no tempo 69 minutos e, neste mesmo instante, a estação 3 inicia o processamento, concluindo a atividade no tempo 97 minutos. O segundo item chegou à estação 3 no tempo 111 minutos, mas seu processamento foi iniciado apenas no tempo 132 minutos. Este evento ocorreu por causa de questões relacionadas com a disponibilidade de equipamentos (Tabela 2). Para o cenário $A$, quando o relógio do simulador chega em 70 minutos, o primeiro item está sendo processado na estação 3. No entanto, um dos pressupostos assumidos durante a construção do modelo propõe que o tempo de reparo comece a ser considerado logo após a desocupação da capacidade da estação 3.

Ao ocorrer a falha a estação 3 fica em reparo por 35 minutos, estando novamente liberada no minuto 132. Neste instante é iniciado o processamento do segundo item, que é finalizado no tempo 160 minutos. Em seguida, inicia-se o processamento do terceiro item, que permaneceu em espera de processo por 7 minutos, entretanto, para o quarto item a ser produzido, não há ocorrência de esperas. No mesmo instante temporal em que o quarto item sai da estação 2, é iniciado seu processamento na estação 3 , contudo, durante o processamento do quarto item, ocorre novamente a falha do equipamento. Após o processamento do quarto item (no tempo 223 minutos) ocorre o tempo de reparo (35 minutos), sendo iniciado o processamento do quinto item no minuto 258. Essa sequência ocorre até a finalizar o processamento dos 10 itens (que compõem 1 Kanban), ocorrendo a cada 70 minutos, em média, a falha do equipamento e posterior à conclusão do processamento, é executado o reparo do equipamento.

As informações referentes às filas ocorridas nas estações 2 e 3, são ilustrativamente apresentadas na Tabela 4.

Tabela 4 - Filas nas estações de trabalho, obtidas com a proposta do cenário A

\begin{tabular}{ccccccccc}
\hline \multirow{2}{*}{ Estação } & \multicolumn{3}{c}{ Tamanho da fila (unidades) } & \multicolumn{3}{c}{ Tempo de espera na fila (minutos) } \\
& Mínimo & Máximo & Média & $\begin{array}{c}\text { Desvio } \\
\text { padrão }\end{array}$ & Mínimo & Máximo & Média & $\begin{array}{c}\text { Desvio } \\
\text { padrão }\end{array}$ \\
\hline 2 & 0 & 5 & 1,89 & 1,504 & 0 & 180 & 90 & 57,542 \\
3 & 0 & 1 & 0,18 & 0,382 & 0 & 21 & 8,40 & 8,758 \\
\hline
\end{tabular}

Fonte: autores (2013). 
O valor obtido para o indicador lead time do contenedor (que diz respeito ao tempo necessário para finalizar a produção de 10 itens) no cenário $A$ foi de 475 minutos com um desvio padrão igual a zero. Com base no valor deste indicador e aplicando a Equação 1 ( $F=0$ e uma demanda igual a 4 contenedores por semana), o número de cartões Kanban necessários para este sistema é de 31 cartões com um desvio padrão igual a zero.

A seguir, inicia-se a análise dos resultados obtidos no cenário B. Além das informações referentes ao 'tempo de início do processamento' e 'tempo de saída', para uma melhor avaliação da variabilidade de fluxo são apresentadas métricas auxiliares. Vale ressaltar que o resultado obtido em cada um dos 10 itens (1 Kanban) foram obtidos com base em 30 rodadas consecutivas no modelo de simulação, representando 9 dias de trabalho de 3 turnos cada. O conceito de cada métrica utilizada é a seguir abordado: 'Mínimo' - mostra o menor valor obtido; 'Máximo' apresenta o maior valor obtido; 'Média' - ilustra o valor médio dos tempos; e 'Desvio padrão' - refere-se ao valor do desvio padrão. Com base nas propostas do cenário $\mathrm{B}$, a Tabela 5 mostra as estatísticas concernentes ao tempo gasto em setup, nas estações 1,2 e 3.

Tabela 5 - Informações estatísticas do tempo gasto em setup. (Tempos em minutos)

\begin{tabular}{cccccc}
\hline \multicolumn{2}{c}{ Tempo gasto em Setup } \\
Estação & Cenário A & \multicolumn{4}{c}{ Cenário B } \\
& Tempo determinístico & Mínimo & Máximo & Média & $\begin{array}{c}\text { Desvio } \\
\text { padrão }\end{array}$ \\
\hline 1 & 5 & 0,06 & 14,93 & 4,81 & 4,13 \\
2 & 2 & 0,10 & 9,82 & 1,49 & 1,93 \\
3 & 7 & 0,39 & 37,30 & 7,33 & 8,55 \\
\hline
\end{tabular}

Fonte: autores (2013).

A partir dos resultados apresentados na Tabela 5, é possível observar a influência o grau médio de variabilidade. Na estação 1 , onde o tempo de setup no cenário A é fixado em 5 minutos. No cenário B este tempo varia dentro de uma faixa de 0,06 até 14,93 minutos, ocorrendo também à condição de variabilidade nas outras duas estações.

Já ao analisar a Tabela 6 , observa-se que a variação do tempo gasto em setup, determinada pelas 30 rodadas do modelo de simulação faz com que a estação 1, inicie o processamento do primeiro na mesma faixa de variação do tempo de setup. Em paralelo a variação do tempo de início de processamento, provocado 
pela operação setup, no cenário B ocorre variação no tempo de processamento natural, conforme proposto nas Tabelas 1 e 2.

Tabela 6 - Informações estatísticas da estação 1 (Tempos em minutos)

\begin{tabular}{ccccccccc}
\hline \multicolumn{1}{c}{ Item } & \multicolumn{1}{c}{ Tempo de início do processamento } \\
& Mínimo & Máximo & Média & $\begin{array}{c}\text { Estação 1 } \\
\text { Desvio } \\
\text { padrão }\end{array}$ & Mínimo & Máximo & Média & $\begin{array}{c}\text { Tempo de saída } \\
\text { padrão }\end{array}$ \\
\hline 1 & 0,06 & 14,93 & 4,81 & 4,13 & 5,90 & 72,08 & 23,24 & 17,44 \\
2 & 5,90 & 72,08 & 23,24 & 17,44 & 16,15 & 127,56 & 45,23 & 25,91 \\
3 & 16,15 & 127,56 & 45,23 & 25,91 & 25,85 & 177,29 & 68,82 & 34,48 \\
4 & 25,85 & 177,29 & 68,82 & 34,48 & 44,05 & 189,25 & 92,98 & 38,80 \\
5 & 44,05 & 189,25 & 92,98 & 38,80 & 48,71 & 211,01 & 116,62 & 46,79 \\
6 & 48,71 & 211,01 & 116,62 & 46,79 & 58,06 & 232,69 & 141,52 & 51,45 \\
7 & 58,06 & 232,69 & 141,52 & 51,45 & 81,67 & 262,51 & 157,10 & 53,64 \\
8 & 81,67 & 262,51 & 157,10 & 53,64 & 107,50 & 274,31 & 172,68 & 51,04 \\
9 & 107,50 & 274,31 & 172,68 & 51,04 & 119,60 & 322,46 & 196,00 & 59,15 \\
10 & 119,60 & 322,46 & 196,00 & 59,15 & 135,32 & 377,51 & 217,51 & 64,55 \\
\hline
\end{tabular}

Fonte: autores (2013).

O resultado da variabilidade provocada pelo setup e pelo tempo de processamento natural faz com que o tempo de saída do primeiro item da estação 1 , oscile dentro de uma faixa que inicia em 5,90 até 72,08 minutos, sendo a média de 23,24 e um desvio padrão de 17,18 minutos. Como existe variabilidade no tempo de saída do primeiro item, essa variação influenciará no tempo de início de processamento do segundo item, e assim sucessivamente até o décimo item.

As flutuações temporais ocorridas na estação 1 provocam na estação 2 a variação no tempo de início de processamento, causando variação no tempo de ociosidade, alterações no padrão da fila, que juntamente com a variabilidade do tempo de processamento natural, provocam variações nos tempos de saída. No que se refere às filas, ao analisar os valores obtidos no cenário $A$ (Tabela 4), é observado que o tamanho máximo da fila na estação 2 é igual a 5 itens e o tempo máximo de espera é igual a 180 minutos com média de 90 e desvio padrão de 57,542 minutos. Já no cenário B (Tabela 9), o tamanho máximo da fila é igual a 8 
itens, sendo o tempo máximo de espera é igual a 440,24 minutos, com média de 128,24 e um desvio padrão de 99,736 minutos. Ao focalizar na estação 2, o teste T fornece como resultados $p=0,765$ quando são comparados os cenários $\mathrm{A}$ e B para o tamanho da fila. No que se refere ao tempo de espera na fila para a estação 2 foi obtido um $p=0,075$. Considerando um nível de significância de $10 \%$ é possível afirmar que ocorrem diferenças significativas entre as médias dos cenários $A$ e $B$ para o indicador tempo de espera na fila para a estação 2, geradas pela ação da variabilidade. Entretanto, com base no resultado obtido pelo teste $\mathrm{T}$ para o indicador tamanho da fila, não é possível rejeitar a hipótese de igualdade entre as médias dos cenários $A$ e $B$. Deste modo, a ação da variabilidade não causou diferenças significativas no tamanho da fila na estação 2 , para o ambiente considerado no modelo de simulação.

Com referência aos tempos de início de processamento e saída da estação 2, a Tabela 7 mostra os resultados alcançados. No cenário $A$, o tempo de ociosidade da estação 2 foi calculado a partir da soma do tempo de setup e o tempo de processamento natural da estação 1 , subtraindo o tempo gasto em setup da estação 2. O valor obtido deste cálculo considerando a ausência de variabilidade foi de 25 minutos. Já no cenário $B$, a ociosidade da estação 2 pode variar entre 5,80 e 62,26 minutos, devido aos possíveis arranjos de posicionamento do tempo de setup e processamento natural, na curva probabilística de cada variável, durante as 30 rodadas do modelo.

Tabela 7 - Informações estatísticas da estação 2 (Tempos em minutos)

\begin{tabular}{ccccccccc}
\hline \multicolumn{1}{c}{ Item } & \multicolumn{7}{c}{ Tempo de início do processamento } & \multicolumn{3}{c}{ Tempo de saída da estação } \\
& Mínimo & Máximo & Média & $\begin{array}{c}\text { Desvio } \\
\text { padrão }\end{array}$ & Mínimo & Máximo & Média & $\begin{array}{c}\text { Desvio } \\
\text { padrão }\end{array}$ \\
\hline 1 & 5,90 & 72,08 & 23,24 & 17,44 & 29,75 & 168,37 & 74,33 & 33,38 \\
2 & 30,57 & 168,37 & 76,77 & 33,71 & 58,67 & 214,77 & 125,56 & 38,34 \\
3 & 58,67 & 214,77 & 127,45 & 38,75 & 73,25 & 285,86 & 175,80 & 46,12 \\
4 & 104,27 & 285,86 & 179,75 & 41,86 & 149,16 & 316,69 & 234,39 & 44,72 \\
5 & 149,16 & 316,69 & 234,39 & 44,72 & 184,31 & 345,78 & 274,47 & 47,08 \\
6 & 184,31 & 345,78 & 274,47 & 47,08 & 205,26 & 424,03 & 328,46 & 53,98 \\
7 & 205,26 & 424,03 & 328,46 & 53,98 & 239,13 & 497,33 & 374,89 & 61,93 \\
8 & 239,13 & 497,33 & 374,89 & 61,93 & 280,53 & 571,74 & 423,95 & 74,94 \\
9 & 280,53 & 571,74 & 423,95 & 74,94 & 320,01 & 604,19 & 470,76 & 78,48 \\
10 & 320,01 & 604,19 & 470,76 & 78,48 & 370,54 & 642,67 & 511,22 & 73,32 \\
\hline
\end{tabular}

Fonte: autores (2013).

A variação no tempo de início de processamento da estação 2, provocado pelo comportamento da estação 1 , juntamente com a variação no tempo de 
processamento natural da estação 2 geram flutuações dos tempos de saída da estação 2. A seguir, os resultados referentes ao comportamento da estação 3 são ilustrados na Tabela 8. Ao avaliar a ociosidade na estação 3 , para o cenário A, essa estação permanece ociosa por 62 minutos (Tabela 3), entretanto, sob condições de variabilidade de fluxo propostas no cenário $B$, a faixa de tempo de ociosidade é compreendida entre 29,36 e 137,60 minutos.

Ao analisar o padrão comportamental das filas na estação 3, os valores obtidos no cenário A (Tabela 4) ilustram um tamanho máximo da fila na igual a 1 itens e o tempo máximo de espera é igual a 21 minutos com média de 8,40 e desvio padrão de 8,75 minutos. Contudo, no cenário B (Tabela 9), o tamanho máximo da fila é igual a 5 itens, sendo o tempo máximo de espera é igual a 434,44 minutos, com média de 54,93 e um desvio padrão de 77,344 minutos. Essas faixas de valores que representam o comportamento das filas foram construídas a partir da posição das variáveis temporais referentes às estações 1 e 2 , onde a variabilidade provocou esse comportamento na estação 3 . No que diz respeito à comparação entre as médias dos cenários A e B para a estação 3 , o teste $\mathrm{T}$ apresenta um valor $p=0,005$ para o tamanho da fila e $p=0,003$ para o tempo de espera na fila. Com base em um nível de significância de $10 \%$ é rejeitada a hipótese nula e afirma-se a existência de diferenças significativas entre as médias dos cenários $A$ e $B$, sendo que as diferenças observadas nas médias dos indicadores tamanho da fila e tempo de espera na fila (estação 3) foram ocasionadas pela ação da variabilidade.

Devido à variação da disponibilidade, conforme apresentado nas Tabelas 1 e 2, juntamente com a variação no tempo natural de processamento da estação e a variabilidade ocorrida nas estações 1 e 2, a estação 3 contribuirá para o aumento da variabilidade do sistema produtivo.

Tabela 8 - Informações estatísticas da estação 3, obtidas com o cenário B (Tempos em minutos)

\begin{tabular}{|c|c|c|c|c|c|c|c|c|}
\hline \multirow{3}{*}{ Item } & \multicolumn{8}{|c|}{$\begin{array}{ll}\text { Estação } 3 \\
\end{array}$} \\
\hline & \multicolumn{4}{|c|}{ Tempo de início do processamento } & \multicolumn{4}{|c|}{ Tempo de saída da estação } \\
\hline & Mínimo & Máximo & Média & $\begin{array}{l}\text { Desvio } \\
\text { padrão }\end{array}$ & Mínimo & Máximo & Média & $\begin{array}{l}\text { Desvio } \\
\text { padrão }\end{array}$ \\
\hline 1 & 29,75 & 174,90 & 84,50 & 42,15 & 41,23 & 204,34 & 114,16 & 53,37 \\
\hline 2 & 58,73 & 246,69 & 140,88 & 48,19 & 66,40 & 294,02 & 167,68 & 57,70 \\
\hline 3 & 73,25 & 331,68 & 205,05 & 56,84 & 97,62 & 346,81 & 235,21 & 61,88 \\
\hline 4 & 171,73 & 409,95 & 266,57 & 63,97 & 181,87 & 431,56 & 295,33 & 57,59 \\
\hline 5 & 217,57 & 515,06 & 317,06 & 58,76 & 220,53 & 520,75 & 350,72 & 63,33 \\
\hline 6 & 231,86 & 520,75 & 384,75 & 65,57 & 288,60 & 676,21 & 422,54 & 86,02 \\
\hline 7 & 299,08 & 686,60 & 451,28 & 90,44 & 314,79 & 708,26 & 479,77 & 89,70 \\
\hline
\end{tabular}




\begin{tabular}{ccccccccc}
\hline 8 & 321,19 & 794,74 & 517,71 & 111,74 & 342,61 & 802,91 & 546,30 & 113,75 \\
9 & 342,61 & 802,91 & 569,72 & 109,75 & 357,25 & 806,69 & 593,47 & 118,30 \\
10 & 414,35 & 813,05 & 605,59 & 115,74 & 447,91 & 821,27 & 631,70 & 111,55 \\
\hline
\end{tabular}

Fonte: autores (2013)

A Tabela 9 apresenta as informações referentes ao comportamento das filas, que ocorreram nas estações 2 e 3.

Tabela 9 - Filas nas estações de trabalho, obtidas com a proposta do cenário B

\begin{tabular}{ccccccccc}
\hline \multirow{2}{*}{ E } & \multicolumn{3}{c}{ Tamanho da fila (unidades) } & \multicolumn{3}{c}{ Tempo de espera na fila (minutos) } \\
stação & Mínimo & Máximo & Média & $\begin{array}{c}\text { Desvio } \\
\text { padrão }\end{array}$ & Mínimo & Máximo & Média & $\begin{array}{c}\text { Desvio } \\
\text { padrão }\end{array}$ \\
\hline 2 & 0 & 8 & 2,03 & 2,056 & 0 & 440,24 & 128,24 & 99,736 \\
3 & 0 & 5 & 0,87 & 1,194 & 0 & 434,44 & 54,93 & 77,344 \\
\hline
\end{tabular}

Fonte: autores (2013).

Uma vez observado o impacto da variabilidade no fluxo do sistema produtivo apresentado na Figura 2, é analisado a seguir o indicador lead time do contenedor. Com base nas condições propostas pelo cenário $B$, o intervalo de tempo necessário para produzir um contenedor Kanban (10 itens) é apresentado na Tabela 10. O resultado do número de cartões Kanban necessários ao sistema produtivo é apresentado na Tabela 11.

Tabela 10 - Leadtime adquirido com o cenário B.

\begin{tabular}{c|c|c|c}
\hline \multicolumn{3}{c}{ Tempo de atravessamento } \\
(minutos) \\
\hline Mínimo & Máximo & Média & $\begin{array}{c}\text { Desvio } \\
\text { padrão }\end{array}$ \\
447,91 & 821,27 & 631,70 & 111,55 \\
\hline
\end{tabular}

Tabela 11 - Necessidade de cartões Kanban.

\begin{tabular}{l|c|c|c}
\hline \multicolumn{4}{c}{ Número de cartões Kanban (unidades) } \\
\hline Mínimo & Máximo & Média & $\begin{array}{c}\text { Desvio } \\
\text { padrão } \\
7,37\end{array}$ \\
29 & 54 & 41,63 & \\
\hline \multicolumn{4}{l}{ Fonte: autores (2013) }
\end{tabular}

No cenário $A$, o valor do lead time do contenedor é de 475 minutos e o número de cartões Kanban determinado é igual a 31 cartões. No entanto, para o cenário $B$, onde todos os coeficientes de variação são menores que 1 , ou seja, um grau médio de variabilidade segundo Hopp e Spearman (2000), em média são necessários 631,70 minutos para produzir um contenedor do Kanban. Nesse caso o desvio padrão do lead time do contenedor foi igual a 111,55 minutos sendo o coeficiente de variação final 0,177 . Comparados os cenários $A$ e $B$, o teste $T$ fornece um $p=0,000$ para o indicador lead time do contenedor, bem como, para o número de cartões Kanban. Para um nível de significância de 10\% é possível afirmar 
que a ação da variabilidade ocasionou diferenças estatisticamente significativas entre as médias dos cenários $\mathrm{A}$ e $\mathrm{B}$, para os indicadores lead time do contenedor $\mathrm{e}$ número de cartões Kanban. Deste modo a hipótese que preconiza a igualdade entre as medidas dos cenários em estudo é rejeitada.

O impacto real da variabilidade de fluxo é observado na Tabela 11, onde a faixa de variação da necessidade de cartões Kanban, é dada pelo intervalo compreendido de 29 até 54 cartões, com média de 41,63 e desvio padrão de 7,37 cartões. As propostas do cenário $\mathrm{B}$, quando comparadas com um ambiente isento de variabilidade, provocaram um aumento médio no número de cartões Kanban de 32\%, causando aumento de work-in-process, gerando necessidade de espaço para disposição, bem como aumento no valor da conta investimentos em matéria-prima. Uma síntese dos resultados obtidos com a execução do teste T é apresentada na Tabela 12.

Tabela 12 - Resultados do teste T

\begin{tabular}{lccccc}
\hline \multirow{2}{*}{ Indicadores } & \multicolumn{2}{c}{ Cenário A } & \multicolumn{2}{c}{ Cenário B } & teste T \\
& Média & Desvio Padrão & Média & Desvio Padrão & $p$-value \\
\hline Lead time do contenedor & 475 & 0 & 631,70 & 111,55 & 0,000 \\
Tamanho da fila (estação 2) & 1,89 & 1,504 & 2,03 & 2,056 & 0,765 \\
Tamanho da fila (estação 3) & 0,18 & 0,382 & 0,87 & 1,194 & 0,005 \\
Tempo de espera (estação 2) & 90,00 & 57,542 & 128,24 & 99,736 & 0,075 \\
Tempo de espera (estação 3) & 8,40 & 8,758 & 54,93 & 77,344 & 0,003 \\
Número de Kanbans & 31 & 0 & 41,63 & 7,37 & 0,000 \\
\hline
\end{tabular}

Fonte: autores (2013).

\section{CONCLUSÕES}

Este artigo teve como principal objetivo, contribuir com o entendimento dos efeitos da Variabilidade de Fluxo sob a ótica da Factory Physics, através do desenvolvimento de um modelo didático que utilizou a abordagem de simulação computacional. Ao realizar uma análise comparativa entre os dois cenários desenvolvidos, foi observado que a variabilidade no sistema produtivo provocou um aumento médio de $32 \%$ no número de cartões Kanban $(p=0,000)$, motivada pelas características do cenário $B$. Mais relevante do que o número em si é a influência significante da variabilidade sobre os resultados analisados. Dentre as principais implicações gerenciais do presente estudo, destaca-se que é evidenciado uma lacuna nos tradicionais modelos matemáticos de dimensionamento de Kanban que não contemplam uma variável referente à variabilidade. Além disso, parece evidente 
que, ao se realizar o dimensionamento de Kanban, além dos pressupostos reconhecidos como itens de demanda elevada e estável no tempo, elevada taxa de qualidade nas operações, e assim por diante, uma ênfase deve ser dada ao aspecto variabilidade dos tempos de processamento das operações; sobretudo, em sistemas produtivos com diversos recursos.

A escolha do dimensionamento do sistema Kanban como forma de ilustrar a influência causada pela variabilidade de fluxo, deu-se pela fragilidade do Kanban frente a ambientes reais de sistemas produtivos com diversas fontes variabilidade. $A$ partir dos resultados obtidos com a construção do modelo didático de simulação, percebeu-se a importância do desenvolvimento de ações que visam à redução da variação temporal existente nos processos produtivos. Desta forma, é possível esperar obter benefícios relativos à simplificação do gerenciamento das atividades e à redução de custos.

\section{AGRADECIMENTOS}

Os autores agradecem ao CNPq, que financiou parte da equipe envolvida nesta pesquisa. Os autores da presente pesquisa agradecem aos revisores pelas importantes contribuições durante o processo de avaliação do artigo, as quais contribuíram sobremaneira para melhorar a versão final dessa pesquisa.

\section{REFERÊNCIAS}

ANTUNES, Junico et al. Sistemas de produção: conceitos e práticas para projeto e gestão da produção enxuta. 1. ed. Porto Alegre: Bookman, 2008.

ANTUNES, José A.V.J. Comparação crítica entre os princípios gerais que regem a teoria das restrições (toc) e a teoria que sustenta a construção dos sistemas de produção com estoque zero (spez): no sentido da construção de uma teoria geral das melhorias no processo. ANANPAD, 1998.

AVELLA-CAMERO,L.;FERNANDEZ-SANCHEZ,E.;VAZQUEZ-ORDAS,C.J. Clasificacion de lãs Estrategias de Fabricacion de las Grandes Empresas Industriales Espanolas. Economıa Industrial, v. 311, p. 77-92, 1996.

BONVIK, Asbjoern M.; GERSHWIN, Stanley B.; TROXEL, Donald E. Operating high variability manufacturing systems. Operation Research Center, MIT, Cambridge, 1995. 
BOYER, K. K.; M. W.LEWIS. Competitive priorities: Investigating the need for tradeoffs in operations strategy. Production and Operations Management, v.11, n.1, p. 9-20, 2002.

BROWNING, T. R.; HEATH, R. D. Reconceptualizing the effects of lean on production costs with evidence from the F-22 program. Journal of Operations Management. v. 27, n. 1, 23-44, 2009.

CALIFE, N. F. S; NOGUEIRA, E; FILHO, A. G. A. Empresas do setor de linha branca e suas estratégias competitivas e de produção. Revista Produção Online, Florianópolis, SC, v.10, n. 2, p. 274-296, jun. 2010.

CORREAA, Henrique L.; GIANESI, Irineu; G.N.; CAON, Mauro. Planejamento, programação e controle da produção. São Paulo: Atlas, 2001.

CHRISTIANSEN,T. ;BERRY, W.L.; BRUUN, P. ;WARD, P. A mapping of competitive priorities, manufacturing practices, and operational performance in groups of Danish manufacturing companies. International Journal of Operations and Production Management, Vol. 23 No. 10, p. 1163-83, 2003.

DANGAYACH,G.S.; DESHMUKH,S.G. Manufacturing strategy: literature review and some issues, International Journal of Operations \& Production Management, Vol. 21 Iss: 7, pp.884 - 932, 2001.

DETTMER, William H. Beyond Lean Manufacturing: Combining Lean and the Theory of Constraints for Higher Performance. Draft, 2001.

FERNANDES, D. C.; SANTOS, F. G.; BARRELLA, W. D.; YOSHIDA, I. C. S. Implantação de células de manufatura na indústria: um estudo de caso. In: ENCONTRO NACIONAL DE ENGENHARIA DE PRODUÇÃO, 21 ENEGEP, 2001, Salvador. Anais...Salvador, 2001.

FERNANDES, F. C. F; GODINHO FILHO, M. Sistemas de coordenação de ordens: revisão, classificação, funcionamento e aplicabilidade. Gestão \& Produção, São Carlos, v.14, n.2, 2007.

HAIR Jr, J. F.; BLACK, W. C.; BABIN, B.; ANDERSON, R. E.; TATHAM, R. L. Análise multivariada de dados. 6 ed. Porto Alegre. Editora Bookman. 2009.

HOPP, Wallace J.; SPEARMAN, Mark L. Factory physics: foundations of manufacturing management. 2. ed. Boston: McGraw-Hill, 2000.

LAGE JUNIOR, Muris; GODINHO FILHO, Moacir. Adaptações ao sistema kanban: revisão, classificação, análise e avaliação. Gestão e Produção, São Carlos, v. 15, n. 1, abr. 2008.

LAW, A. M.; KELTON, W. David. Simulation modeling and analysis. 3th ed. [S.L.]: McGraw-Hill, 2000. 
MARODIN, G; DE ECKERT, C. P; SAURIN, T. A. Avançando na implantação da logística interna lean: dificuldades e resultados alcançados no caso de uma empresa montadora de veículos. Revista Produção Online, Florianópolis, SC, v.12, n. 2, p. 455-479, abr./jun. 2012.

OHNO, Taiichi. O Sistema toyota de produção: além da produção em larga escala. Porto Alegre: Bookman, 1997.

PACHECO, D. A. J. Integrando a Estratégia de Produção com a Teoria das Restrições, Lean e Seis Sigma: uma abordagem metodológica. Dissertação de Mestrado. Programa de Pós Graduação em Engenharia de Produção e Sistemas, UNISINOS, São Leopoldo, 2012.

PERGHER, I.; RODRIGUES, L. H.; LACERDA, D. P. Discussão teórica sobre o conceito de perdas do Sistema Toyota de Produção: inserindo a lógica do ganho da Teoria das Restrições. Gestão e Produção, v.18, n. 4, p. 673-686, 2011.

PETTERSEN, Arne; SEGERSTEDT, Anders. Restricted work-in-process: a study of differences between Kanban and CONWIP. International Journal of Production Economics, 2009.

PONTES, H. L. J; PALMA, J. G; PORTO, A. J. V. Simulinve- Um simulador de inventário para um centro de distribuição de peças. Revista Produção Online, Florianópolis, SC, v.3, n. 3, 2008.

RABBANI, M.; LAYEGH, J.; EBRAHIM, M. R. Determination of number of kanbans in a supply chain system via Memetic algorithm. Advances in Engineering Software, 2009.

SANDRINI, Luiz Guilherme; MESQUITA, Marco Aurélio. O trade-off entre a folga na capacidade de produção e o nível de estoque. Revista Gestão Industrial, 2008.

SANDANAYAKE, Y.G.; ODUOZA, C.F.; PROVERBS, D.G. A systematic modelling and simulation approach for JIT performance optimisation. Robotics and Computer Integrated Manufacturing, 2008.

SHAHABUDEEN, P.; SIVAKUMAR, G.D. Algorithm for the design of single-stage adaptive kanban system. Computers \& Industrial Engineering, 2008.

SHINGO, Shigeo. O Sistema Toyota de produção: do ponto de vista de engenharia de produção. 1. ed. Porto Alegre: Bookman, 1996.

SLACK, Nigel; CHAMBERS, Stuart; JOHNSTON, Robert. Administração da produção. 2. ed. São Paulo: Atlas, 2009.

SLACK, Nigel et al. Administração da produção. 1. ed. São Paulo: Atlas, 1997. 
SUWANRUJI, P.; ENNS, S. T. Evaluating the effects of capacity constraints and demand patterns on supply chain replenishment strategies. International Journal of Production Research, 2006.

TUBINO, D. F.; LEMOS, A. C. D. Aplicação de uma metodologia de ajuste do sistema kanban em um caso real utilizando a simulação computacional. In:

ENCONTRO NACIONAL DE ENGENHARIA DE PRODUÇÃO, 19 ENEGEP, 1999, Rio de Janeiro. Anais... Rio de Janeiro, 1999.

WHITE, R. E.; PRYBUTOK, V. The Relationship Between JIT practices and Type of production system. Omega, The International Journal of Management Science, $v$. 29, n. 2, p. 113-124, 2001.

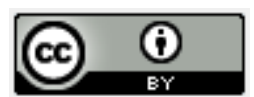

Artigo recebido em 06/03/2013 e aceito para publicação em 24/07/2013.

DOI: http://dx.doi.org/10.14488/1676-1901.v14.i1.1542 